\title{
A Lunar Communications and Navigation Satellite Concept for the Robotic Lunar Exploration Program
}

\author{
Jeffrey J. Gramling, Yi-Pheng Ngan, David A. Quinn and David C. Folta \\ National Aeronautics and Space Administration \\ Goddard Space Flight Center \\ Greenbelt Maryland \\ Bruce E. LeRoy \\ The Aerospace Corporation \\ 1000 Wilson Blvd, Suite 2600 \\ Arlington Virginia
}

The Second Robotic Lunar Exploration Program (RLEP) mission has two primary objectives. Broadly stated, they are "To See the Light" and "To Touch the Ice", meaning one mission objective is to survey the lighting conditions on the rim of a candidate crater over the course of a year, and the second objective is to descend into this crater to search for water ice. The Space Communications Program Office at NASA/GSFC is supporting the RLEP-2 Project by developing a communications and navigation architecture for the mission.

The primary candidate crater studied is the Shackleton Crater near the South Pole of the Moon. The rim of this crater has approximately 14 contiguous days of visibility to the Earth over the course of a month, while mission elements in the crater have no line of sight to either the Earth or potentially an element on the rim of the crater. One solution studied is to launch a relay spacecraft with RLEP-2 to provide communications and navigation services to the elements on the lunar surface.

To support operations concepts requiring long duration contacts between the relay and the elements in the crater, an inclined elliptical orbit originally conceived by Todd Ely at JPL was chosen for the relay. The orbit was adjusted to lower the maximum range while maintaining its "frozen" nature, meaning once placed in this orbit little delta- $V$ is required for orbit maintenance. The orbital parameters were adjusted such that the relay has visibility into the crater for approximately 8.7 hours of the 12 hour orbital period.

The relay satellite design draws heavily upon heritage concepts and hardware to achieve a very low risk implementation. The design concept is a 3-axis spacecraft that is capable of supporting return communication links from multiple surface elements concurrently through a four-element S-Band Multiple Access phased array with two forward links provided through single dedicated elements. The payload is a bent-pipe relay with beamforming performed on the ground (Earth).

To mitigate concerns with multipath observed during Mars missions, the preliminary design employs PN-spread signal structures which have been used successfully by Tracking and Data Relay Satellite System (TDRSS) users for simultaneous real-time and recorded data playback. Details of the relay communication trade studies and rationale for the selected design elements are discussed in the paper. 
Navigation for surface elements is provided primarily through coherent two-way range and Doppler. Where this method is insufficient due to the motion of the element or due to more accurate location knowledge requirements, radiometric tracking is augmented with celestial navigation or triangulation using a landed beacon or other signal source with a known location. Preliminary navigation architecture and analyses results are addressed in the paper.

The frequency plan for the link between the relay and the Earth is an area where further study is warranted. Where data rates are not a driving requirement, S-Band is a suitable choice for Earth-lunar transit, lunar orbit insertion, and descent and landing. The preliminary relay design employs a Ku-Band link to avoid reverse banding of S-Band system. Further work must also be performed to develop a plan for ground station support. To ensure wise investment in ground infrastructure development, this plan should be extensible and consistent with preliminary Constellation architectures.

This paper details the design and operations trades that led to the preliminary design of the relay satellite. Estimates of the relay weight, power, and communications, ranging, and Doppler performance are presented. 